\title{
SCHUNCK CLASSES AND PROJECTORS IN A CLASS OF LOCALLY FINITE GROUPS
}

\author{
by M. J. TOMKINSON
}

(Received 18th April 1994)

\begin{abstract}
We introduce a definition of a Schunck class of periodic abelian-by-finite soluble groups using major subgroups in place of the maximal subgroups used in finite groups. This allows us to develop the theory as in the finite case proving the existence and conjugacy of projectors. Saturated formations are examples of Schunck classes and we are also able to obtain an infinite version of Gaschütz $\Omega$-subgroups.
\end{abstract}

1991 Mathematics Subject Classification: 20F50, 20D10

\section{Introduction}

The generalization of Sylow and Hall subgroups in finite soluble groups led to the definition of saturated formations and their associated projectors by Gaschütz [3] in 1963. These were then extended further to Schunck classes and their projectors by Schunck [8] in 1967. An account of this theory is given in Chapter III of [1]. For locally finite-soluble groups there has been a number of successful extensions of the theory of formations; in particular, it was shown in [2] that this could be carried out in classes with a good Sylow theory including periodic soluble linear groups and periodic almost locally nilpotent groups.

To date there has been no attempt to extend the idea of a Schunck class to infinite groups. The main reason is that the definition of a Schunck class involves the primitive homomorphic images of finite soluble groups which are related to their maximal subgroups. In infinite groups there are usually insufficient maximal subgroups and if a maximal subgroup has infinite index it is not clear that the corresponding homomorphic image will behave in any way like a finite primitive soluble group.

Here we suggest a possible approach replacing maximal subgroups by the major subgroups which we introduced in [9]. We work within the class $\mathfrak{U} \mathcal{S}^{*}$ of periodic soluble abelian-by-finite groups. We showed in [10] that the major subgroups work particularly well in the class of nilpotent-by-finite groups and it seems possible that our results could be extended to that class; however we shall see that the almost abelian condition is required for other aspects of our proof.

We begin by recalling the definition and basic properties of major subgroups of $\mathfrak{U S}^{*}$ groups and introducing semiprimitive Chernikov groups which will be our infinite analogue of primitive finite soluble groups. In Section 3 we define a Schunck class $\mathfrak{X}$ of 
$\mathscr{A} \Im^{*}$-groups and prove the main result, that each $\mathfrak{A} \Im^{*}$-group has a single conjugacy class of $\mathfrak{X}$-projectors.

We see that saturated formations of $\mathfrak{1 S}^{*}$-groups are examples of Schunck classes and that we can also define Gaschütz $\Omega$-subgroups (see $[1$, p. 311] or $[4$, p. 23]). This latter example does generalize the results in the finite case but requires a definition which is rather more complicated than the original finite version.

\section{Major subgroups and semiprimitive Chernikov groups}

Let $U$ be a subgroup of the group $G$ and consider the properly ascending chains

$$
U=U_{0}<U_{1}<\cdots<U_{\alpha}=G
$$

from $U$ to $G$. We define $m(U)$ to be the least upper bound of the types $\alpha$ of all such chains. Thus $m(U)$ is a measure of how far from $G$ the subgroup $U$ is; $m(U)=1$ if and only if $U$ is a maximal subgroup of $G$. A proper subgroup $M$ of $G$ is said to be a major subgroup if $m(U)=m(M)$, whenever $M \leqslant U<G$. The intersection of all major subgroups of $G$ is denoted by $\mu(G)$, which coincides with the Frattini subgroup $\varphi(G)$ if $G$ is finitely generated.

Proposition 2.1. [9, Lemma 2.3]. Every proper subgroup of a group $G$ is contained in a major subgroup of $G$.

In [10], we considered the major subgroups of nilpotent-by-finite groups and obtained the following result.

Proposition 2.2. [10] Let $A$ be a nilpotent normal subgroup of finite index in $G$.

(i) If $M$ is a major subgroup of $G$, then $M \geqslant A^{\prime}$ and $G / M_{G}$ is a Chernikov group.

(ii) If $M$ is a non-maximal major subgroup of $G$, then $A / M_{G}$ is a divisible Chernikov p-group, for some prime $p$, and is divisibly irreducible as a $\mathbb{Z}(G / A)$-module.

As usual, a divisible abelian $p$-group $A$ which is a $\mathbb{Z} G$-module for some group $G$ is said to be divisibly irreducible if every submodule of $A$ is finite (see [5], for example).

We use the notation $M_{G}$ to denote the core of $M$ in $G$. A finite soluble group $G$ is primitive if it contains a maximal subgroup $M$ such that $M_{G}=1$. Then the Fitting subgroup of $G$ is the unique minimal normal subgroup $A$ of $G$ and the complements of $A$ in $G$ are the conjugates of $M$. As a $\mathbb{Z} M$-module, $A$ is faithful and irreducible. The terminology comes from the theory of permutation groups; a finite soluble group $G$ is primitive if and only if it has a faithful primitive permutation representation (on the cosets of $M$ ).

Corresponding to this we define a semiprimitive group $G$ to be the split extension of a faithful divisibly irreducible $\mathbb{Z} M$-module $D$ by the finite soluble group $M$. In such a group, $M$ is a major subgroup of $G$ and $M_{G}=1$. An infinite soluble Chernikov group $G$ 
is semiprimitive if and only if $G$ has a transitive faithful permutation representation in which all the non-trivial $G$-blocks are finite.

Proposition 2.3. With the notation above, let $G=D M$ be a semiprimitive Chernikov group. Then

(i) $D=\operatorname{Fit}(G)$,

(ii) $G / \Omega_{n}(D) \cong G$.

Proof. (i) Suppose $N$ is a normal nilpotent subgroup of $G$. Then $N D$ is nilpotent and so $D \leqslant Z(N D)$. Therefore $N D \cap M \triangleleft D M=G$ and so $N D \cap M=1$. It follows that $N D=N D \cap M D=(N D \cap M) D=D$ and so $N \leqslant D$.

(ii) Writing $G=D M$, consider the map $\varphi: G \rightarrow G$ defined by $(d m) \varphi=d^{p} m$.

We have

$$
\left(\left(d_{1} m_{1}\right)\left(d_{2} m_{2}\right)\right) \varphi=\left(d_{1} d_{2}^{m-1} m_{1} m_{2}\right) \varphi=d_{1}^{p}\left(d_{2}^{p}\right)^{m_{1}^{-1}} m_{1} m_{2}=d_{1}^{p} m_{1} d_{2}^{p} m_{2}
$$

and so $\varphi$ is a homomorphism. Since $D$ is divisible, $\varphi$ is surjective and clearly ker $\varphi=\Omega_{1}(D)$. Thus $G / \Omega_{1}(D) \cong G$ and, by induction, $G / \Omega_{n}(D) \cong G$.

\section{Schunck classes and projectors}

Let $\mathfrak{X}$ be a class of groups and $X$ a subgroup of $G$. Then $X$ is an $\mathfrak{X}$-projector of $G$ if $X N / N$ is a maximal $\mathfrak{X}$-subgroup of $G / N$, for each $N \triangleleft G$.

We consider what conditions on $\mathfrak{X}$ are needed to ensure that every $\mathfrak{A} \mathcal{S}^{*}$-group has $\mathfrak{X}$ projectors. The main problems concern the semiprimitive Chernikov groups.

Lemma 3.1. Let $G$ be an infinite semiprimitive Chernikov group and let $\mathfrak{X}$ be a $Q$-closed class of groups such that $G$ has an $\mathfrak{X}$-projector $X$. If $G$ is the union of an ascending chain of $\mathfrak{X}$-subgroups then $\mathrm{G}$ is an $\mathfrak{X}$-group.

Proof. Let $G=D M=\bigcup_{i=1}^{\infty} H_{i}$, with $H_{i} \in \mathfrak{X}$. There is an integer $k$ such that $M \leqslant H_{k}$ and so $D H_{i}=G$, for all $i \geqslant k$. In particular $G / D \in \mathfrak{X}$ and so $D X=G$.

Suppose, if possible, that $X \neq G$. Then $D \cap X$ is a proper normal subgroup of $G$ and the hypotheses of the lemma pass to $G / D \cap X$. We may therefore assume that $D \cap X=1$ and so $X$ is finite.

For some $i \geqslant k$, write $K=D \cap H_{i} \triangleleft D H_{i}=G$. Then $H_{i} / K$ and $X K / K$ are complements to $D / K$ in $G / K$. Since $D / K$ is abelian, there is an automorphism $\varphi$ of $G / K$ mapping $H_{i} / K$ to $X K / K$ [6, Hilfssatz VI.7.14]. But $H_{i} / K<H_{i+1} / K$ and so $X K / K<\left(H_{i+1} / K\right)^{\varphi}$, contrary to $X K / K$ being a maximal $\mathfrak{X}$-subgroup of $G / K$. This contradiction shows that $X=G$ and so $G \in \mathfrak{X}$.

It follows from this lemma that if we are to define a Schunck class $\mathfrak{X}$ of $\mathscr{\mathfrak { A }} \mathcal{S}^{*}$-groups so that every $\mathfrak{U S}^{*}$-group has $\mathfrak{X}$-projectors we need a condition that every semiprimitive group that is a union of $\mathfrak{X}$-groups is also an $\mathfrak{X}$-group. Without imposing a condition of 
this type we could take $\mathfrak{X}$ to be the class of all finite soluble groups when it is clear that an infinite periodic abelian group does not even possess maximal $\mathfrak{X}$-subgroups.

Our aim is to define Schunck classes by concentrating on the finite primitive and infinite semiprimitive homomorphic images of a group so that $G \in \mathfrak{X}$ if and only if all its primitive and semiprimitive images are in $\mathfrak{X}$. A further problem can arise here since a straightforward definition along these lines would allow us to consider the single primitive group $C_{2}$ and the semiprimitive group $H=C_{3^{\infty}} \rtimes C_{2}$ in which an element of order 2 inverts every 3 -element. We could define a group $G$ to be an $\mathfrak{X}$-group if these are its only primitive and semiprimitive images. But in the group

$$
G=\left(\begin{array}{c}
\infty \\
D r \\
n=1
\end{array} C_{3^{n}}\right) \rtimes C_{2},
$$

in which an element of order 2 inverts every 3 -element, the only $\mathfrak{X}$-subgroups are of order 2, whereas $G$ has a homomorphic image isomorphic to $H$ so that $G$ does not have an $\mathfrak{X}$-projector. The difficulty here is caused by $H$ not being the union of finite $\mathfrak{X}$ groups. We therefore include this in our definition.

Definition 3.2. A Schunck class of $\mathfrak{U}^{*}$-groups is a subclass $\mathfrak{X}$ of $\mathscr{\mathfrak { A }} \Im^{*}$ satisfying the following conditions

(S1) $Q \mathfrak{X}=\mathfrak{X}$,

(S2) an infinite semiprimitive Chernikov group $H$ is an $\mathfrak{X}$-group if and only if $H$ is the union of an ascending chain of finite $\mathfrak{X}$-groups,

(S3) if $G$ is an $\mathfrak{U}^{*}$-group such that every finite primitive image and every infinite semiprimitive image is an $\mathfrak{X}$-group, then $G$ is an $\mathfrak{X}$-group.

It should be noted that the class of finite $\mathfrak{X}$-groups forms a Schunck class of finite soluble groups and so our results will, in fact, be generalizations of the "classical" results for finite soluble groups.

Although we do not use the result in its general form, the condition (S2) leads easily to a local closure property of $\mathfrak{X}$.

Lemma 3.3. Let $\mathfrak{x}$ be a Schunck class of $\mathfrak{U S}^{*}$-groups. Then

$$
L \mathfrak{X} \cap \mathscr{U}^{*}=\mathfrak{X} .
$$

In particular, an $\mathfrak{X}$-subgroup of an $\mathfrak{U}^{*}$-group is contained in a maximal $\mathfrak{X}$-subgroup.

Proof. Let $G \in L \mathfrak{X} \cap \mathfrak{A} \mathcal{S}^{*}$; if $G \notin \mathfrak{X}$ then it has a finite primitive image $G / K \notin \mathfrak{X}$ or an infinite semiprimitive image $G / L \notin \mathfrak{X}$. But $G$ has an $\mathfrak{X}$-subgroup $F$ such that $K F=G$ and so $G / K \cong F / F \cap K \in Q \mathfrak{X}=\mathfrak{X}$. Also $G / L \in Q L \mathfrak{X}=L \mathfrak{X}$ and so $G / L$ is the union of an ascending chain of $\mathfrak{X}$-subgroups. It follows from (S2) that $G / L \in \mathfrak{X}$.

If $M$ is a major subgroup of the $\mathscr{U} \Theta^{*}$-group $G$ then $G / M_{G}$ is either a finite primitive 
group or an infinite semiprimitive group. It is clear therefore that $\mu(G)$ is the intersection of all $L \triangleleft G$ such that $G / L$ is either finite primitive or infinite semiprimitive. The "saturation property" for $\mathfrak{X}$ follows immediately.

Lemma 3.4. Let $\mathfrak{X}$ be a Schunck class of $\mathfrak{M} \subseteq^{*}$-groups and let $G \in \mathfrak{H} \subseteq^{*}$. Then $G \in \mathfrak{X}$ if and only if $G / \mu(G) \in \mathfrak{X}$.

\section{4. $\mathfrak{x}$-projectors}

Having obtained the elementary properties of the Schunck class $\mathfrak{X}$ we can now prove the main theorem. Although proof is based on the approach given by Gaschütz [4], it will be seen that the induction arguments used in the finite case require considerable modification.

Throughout this section $\mathfrak{X}$ denotes a Schunck class of $\mathscr{\mathscr { C }} \mathfrak{S}^{*}$-groups.

Lemma 4.1. Let $A$ be an abelian normal subgroup of the $2 \mathcal{H}^{*}$-group $G$ and let $X$ be an $\mathfrak{X}$-subgroup of $G$ such that $A X=G$ and $G / A \cap X \in \mathfrak{X}$. Then $G$ is an $\mathfrak{X}$-group.

Proof. If $G \notin \mathfrak{X}$ then $G$ has a finite primitive or infinite semiprimitve image $G / K \notin \mathfrak{X}$. Since $G / A \cap X \in \mathfrak{X}, K \ngtr A \cap X$. Therefore $K<(A \cap X) K \leqslant A K$. If $(A \cap X) K=A K$, then $X K=X A K=G$ so that $G / K \cong X / X \cap K$ contrary to $G / K \notin \mathfrak{X}$. Therefore we may assume that

$$
K<(A \cap X) K<A K .
$$

(a) $G / K$ finite primitive. In this case $(A \cap X) K / K$ and $A K / K$ are nontrivial abelian normal subgroups of $G / K$ and so are equal (both being $\mathrm{Fit}(G / K)$ ) contrary to (*).

(b) $G / K$ infinite semiprimitive. In this case

$$
K<(A \cap X) K<A K \leqslant D,
$$

where $D / K=D(G / K)=$ Fit $(G / K),(2.3(i))$.

Since $D / K$ is divisibly irreducible, $(A \cap X) K / K$ is finite and so $(A \cap X) K \leqslant D_{n}$, for some $n$, where $D_{n} / K=\Omega_{n}(D / K)$. Since $G / A \cap X \in \mathfrak{X}$, this shows that $G / D_{n} \in \mathfrak{X}$ and so, by Lemma 2.3(ii), $G / K \in \mathfrak{X}$. This final contradiction shows that $G$ must be an $\mathfrak{X}$-group.

Lemma 4.2. Let $A$ be an abelian subgroup of the $\mathcal{Y G}^{*}$-group $G$ such that $G / A$ is a finite $\mathfrak{X}$-group. Then

(i) $G$ has an $\mathfrak{X}$-subgroup $X$ such that $A S X=G$,

(ii) if $X$ and $Y$ are maximal $X$-subgroups of $G$ such that $A X=A Y=G$, then $X$ and $Y$ are conjugate in $G$.

Proof. (i) There is a finite subgroup $F$ of $G$ such that $A F=G$. Thus $F / A \cap F \in \mathbb{X}$ and, 
by Lemma II.11 of [3], there is an $\mathfrak{X}$-subgroup $X$ of $F$ such that $(A \cap F) X=F$. Hence $A X=A F=G$.

(ii) Since $A$ is abelian, $A \cap X \triangleleft A X=G$ and, similarly, $A \cap Y \triangleleft G$. Hence $M=(A \cap X)(A \cap Y) \triangleleft G$.

Let $U=\langle X, Y\rangle$; then $U / M$ is generated by the subgroups $M X / M$ and $M Y / M$. But

$$
M X \cap A=X(A \cap Y) \cap A=(A \cap X)(A \cap Y)=M
$$

so $M X / M \cong G / A$ is finite. Hence $U / M$ is finite and we prove the result by induction on $|U / M|$.

First, suppose $U / M \in \mathfrak{X}$. The group $U / A \cap X$ has an $\mathfrak{X}$-subgroup $(A \cap X) Y /(A \cap X)$ and an abelian normal subgroup $A \cap U / A \cap X$. Since $(A \cap U) \cap(A \cap X) Y=M$, we can apply Lemma 4.1 to deduce that $U / A \cap X \in \mathfrak{X}$. But now $U$ has an $\mathfrak{X}$-subgroup $X$ and an abelian normal subgroup $A \cap U$ satisfying the hypotheses of Lemma 4.1 and so $U \in \mathfrak{X}$. But $U=\langle X, Y\rangle$ and $X, Y$ are maximal $\mathfrak{X}$-subgroups of $G$, so $U=X=Y$. We may therefore assume that $U / M \notin X$.

Then $U / M$ has a finite primitive homomorphic image $U / K \notin \mathfrak{X}$. Since $U / A \cap U \in \mathfrak{X}$, $K \ngtr A \cap U$ and so $(A \cap U) K / K=\operatorname{Fit}(U / K)$. Since $K X / K$ and $K Y / K$ are $\mathfrak{X}$-groups, they are complements to $\operatorname{Fit}(U / K)$ in $U / K$ and so are conjugate by an element of $\operatorname{Fit}(U / K)$. That is, there is an element $a \in A$, such that $(K Y)^{a}=K X$. Consider the subgroups $X$ and $Y^{a}$. Clearly $A \cap Y^{a}=A \cap Y$ and so $(A \cap X)\left(A \cap Y^{a}\right)=M$ but $\left\langle X, Y^{a}\right\rangle \leqslant K X<U$. Thus $\left|\left\langle X, Y^{a}\right\rangle:(A \cap X)\left(A \cap Y^{a}\right)\right|<|U / M|$ and, by induction, $X$ and $Y^{a}$ are conjugate. Hence $X$ and $Y$ are conjugate.

It was suggested in the Introduction that it might be possible to develop this theory in almost nilpotent groups. However the above proof relies heavily on the opening remark that $A \cap X \triangleleft A X=G$ and we have been unable to produce a satisfactory version of this lemma for nilpotent-by-finite groups. This lemma is used in showing that the $\mathfrak{X}$ subgroups obtained do, in fact, cover all $\mathfrak{X}$-images of $G$.

Lemma 4.3. Let $A$ be an abelian normal subgroup of the $\mathfrak{U}^{*}$-group $G$ with $G / A$ a finite $\mathfrak{X}$-group and let $X$ be a maximal $\mathfrak{X}$-subgroup of $G$ such that $A X=G$.

If $N \leqslant A$ with $N \triangleleft G$ and $G / N \in \mathfrak{X}$, then $N X=G$.

Proof. Suppose $N X \neq G$. Then $A \cap N X \triangleleft A X=G$ and $A / A \cap N X$ is a nontrivial abelian normal subgroup of $G / A \cap N X$.

If $A / A \cap N X$ has a nontrivial finite image then there is a subgroup $B \triangleleft G$ with $N(A \cap X)=A \cap N X \leqslant B<A$ and $G / B$ a finite $\mathfrak{X}$-group.

By Lemma 4.2, $G$ has a maximal $\mathfrak{X}$-subgroup $Y$ such that $B Y=G$ and hence $A Y=G$. By Lemma 4.2(ii), $Y$ and $X$ are conjugate and hence $B X=G$. But this is contrary to $A \cap B X=B(A \cap X)=B<A$.

So we may assume that $A / A \cap N X$ is divisible and so there is a subgroup $B \triangleleft G$ with $A \cap N X \leqslant B<A$ and $G / B$ a Chernikov $\mathfrak{X}$-group. There is then a subgroup $K \triangleleft G$ with 
$B \leqslant K$ and $G / K$ an infinite semiprimitive $\mathfrak{X}$-group. By condition (S2), $G / K$ is the union of an ascending chain of finite $\mathfrak{X}$-subgroups $H_{i} / K$.

We show that $H_{i} / A \cap K \in \mathfrak{X}$. Since $G / K$ is semiprimitive, there is a finite subgroup $V / K$ such that $G / K=(A K / K) \rtimes(V / K)$ and integer $k$ such that $V \leqslant H_{i}$, for all $i \geqslant k$. Now $A \cap K=A \cap V$ and so $V / A \cap K \cong G / A \in \mathfrak{X}$. Let $W / A \cap K$ be a maximal $\mathfrak{X}$-subgroup of $H_{i} / A \cap K$ containing $V / A \cap K$; then $\left(A \cap H_{i}\right) W=A V \cap H_{i}=H_{i}$ and so $W / A \cap K$ is an $x$-projector of $H_{i} / A \cap K$ [3, Corollary II.13]. It follows that $K W=H_{i}$; but $W \geqslant V \geqslant K$ and so $W=H_{i}$ and $H_{i} / A \cap K \in \mathfrak{X}$, as required.

Since $B X / B$ is finite, there is an integer $m$ such that $X \leqslant H_{i}$ and $H_{i} / A \cap K \in \mathfrak{X}$, for all $i \geqslant m$. By Lemma $4.2, H_{i}$ has a maximal $\mathfrak{X}$-subgroup $S$ such that $(A \cap K) S=H_{i}$ and hence $\left(A \cap H_{i}\right) S=H_{i}$. Also $\left(A \cap H_{i}\right) X=H_{i}$ and so, by Lemma 4.2(ii), $S$ and $X$ are conjugate in $H_{i}$. It follows that $(A \cap K) X=H_{i}$ and hence $K X=H_{i}$, for all $i \geqslant m$. This is contrary to $K X / X$ being finite and so we must have $N X=G$.

Lemma 4.4. Let $A$ be a normal abelian subgroup of the $\mathfrak{1} \mathcal{S}^{*}$-group $G$ such that $G / A$ is a finite $\mathfrak{X}$-group and let $X$ be a maximal $\mathfrak{X}$-subgroup of $G$ such that $A X=G$.

If $N \triangleleft G$ then $N X / N$ is a maximal $\mathfrak{X}$-subgroup of $G / N$.

Proof. (1) $N \leqslant A$.

Suppose $N X \leqslant X^{*}$ with $X^{*} / N \in \mathfrak{X}$. Applying Lemma 4.3 in the group $X^{*}$ noting that $\left(A \cap X^{*}\right) X=X^{*}$, we obtain $N X=X^{*}$ and so $N X / N$ is a maximal $\mathfrak{X}$-subgroup of $G / N$.

(2) General case Now $N \cap A \triangleleft G$ and, by case (1), $(N \cap A) X / N \cap A$ is a maximal $\mathfrak{X}$ subgroup of $G / N \cap A$. Thus we may assume that $N \cap A=1$ and so $N$ is finite.

By induction, we may assume that $N$ is a minimal normal subgroup of $G$ and so is abelian. Since $N \cap A=1$, it follows that $A N$ is an abelian normal subgroup of $G$ containing $N$. We can therefore apply case (1) again with $A N$ replacing $A$.

Theorem 4.5. Let $\mathfrak{X}$ be a Schunck class of $\mathfrak{A} \varsigma^{*}$-groups; then the $\mathfrak{A} \subseteq^{*}$-group $G$ has $\mathfrak{X}$-projectors and any two $\mathfrak{X}$-projectors of $G$ are conjugate in $G$.

Proof. Let $A$ be an abelian normal subgroup of finite index in $G$. By the finite case, $G / A$ has an $\mathfrak{X}$-projector $\bar{X} / A$. By Lemma $4.2(i), \bar{X}$ has a maximal $\mathfrak{X}$-subgroup $X$ such that $A X=\bar{X}$.

Let $N \triangleleft G$; we show that $N X / N$ is a maximal $\mathfrak{X}$-subgroup of $G / N$. Applying Lemma 4.4 to the normal subgroup $N \cap \bar{X}$ of $\bar{X}$, we see that $(N \cap \bar{X}) X /(N \cap \bar{X})$ is a maximal $\mathfrak{X}$-subgroup of $\bar{X} / N \cap \bar{X}$. The isomorphism between $N \bar{X} / N$ and $\bar{X} / N \cap \bar{X}$ shows that $N X / N$ is a maximal $\mathfrak{E}$-subgroup of $N \bar{X} / N$.

Suppose that $N X \leqslant X^{*}$ with $X^{*} / N \in \mathfrak{X}$. Since $N \bar{X} / A N$ is a maximal $\mathfrak{X}$-subgroup of $G / A N$ and $N \bar{X}=A N X \leqslant A X^{*}$ we have $N \bar{X}=A X^{*}$ and so $X^{*} \leqslant N \bar{X}$. But $N X / N$ is a maximal $\mathfrak{X}$-subgroup of $N \bar{X} / N$ and so $N X=X^{*}$ and $N X / N$ is a maximal $\mathfrak{X}$-subgroup of $G / N$. Thus $X$ is an $\mathfrak{X}$-projector of $G$.

If $X$ and $Y$ are $\mathfrak{X}$-projectors of $G$, then $A X / A$ and $A Y / A$ are $\mathfrak{X}$-projectors of $G / A$ and so are conjugate in $G / A$. We may therefore assume that $A X=A Y$. Now it follows from Lemma 4.2(ii) that $X$ and $Y$ are conjugate. 
Corollary 4.6. Let $G \in \mathfrak{U} \Im^{*}$ and $N \triangleleft G$. Then the $\mathfrak{X}$-projectors of $G / N$ are the subgroups $N X / N$, where $X$ is an $\mathfrak{X}$-projector of $G$.

Proof. It is clear that if $X$ is an $\mathfrak{X}$-projector of $G$ then $N X / N$ is an $\mathfrak{X}$-projector of $G / N$.

Let $\bar{X} / N$ be an $\mathfrak{X}$-projector of $G / N$. Then $\bar{X} / N$ is conjugate to $N X / N$ and so $\bar{X}=N X^{g}$ and $X^{g}$ is an $\mathfrak{X}$-projector of $G$.

The proof of Theorem 4.5 actually contains the following useful fact.

Lemma 4.7. Let $A$ be a normal abelian subgroup of finite index in the $\mathfrak{A S}^{*}$-group $G$ and let $\bar{X} / A$ be an $\mathfrak{X}$-projector of $G / A$. If $X$ is a maximal $\mathfrak{X}$-subgroup of $\bar{X}$ such that $A X=\bar{X}$, then $X$ is an $\mathfrak{X}$-projector of $G$.

Corollary 4.8. Let $X$ be an $\mathfrak{X}$-projector of the $\mathfrak{A} \Im^{*}$-group $G$ and let $L$ be a subgroup of $G$ containing $X$. Then $X$ is an $\mathfrak{X}$-projector of $L$.

Proof. Let $A$ be a normal abelian subgroup of finite index in $G$. Then $A X / A$ is an $\mathfrak{X}$-projector of $G / A$. By the finite case, $A X / A$ is an $\mathfrak{X}$-projector of $A L / A$ and hence $(A \cap L) X /(A \cap L)$ is an $\mathfrak{X}$-projector of $L / A \cap L$. The result now follows from Lemma 4.7.

It follows from Corollary 4.8 that the $\mathfrak{X}$-projectors of an $\mathfrak{A} \mathcal{S}^{*}$-group $G$ can also be characterised as the $\mathfrak{X}$-covering subgroups of $G$, as in the finite soluble case.

\section{Examples}

\subsection{Saturated formations}

Let $\pi$ be a set of primes and, for each $p \in \pi$, let $f(p)$ denote a formation of finite soluble groups. The saturated formation $\mathfrak{F}$ of $\mathfrak{A S}^{*}$-groups defined by $\mathfrak{f}$ is

$$
\mathfrak{F}=\mathcal{Q}_{\pi} \mathfrak{S}_{\pi}^{*} \cap \bigcap_{p \in \pi} \mathfrak{S}_{p^{\prime}} \mathfrak{S}_{p} \mathfrak{f}(p)
$$

An $2 S^{*}$-group $G$ is an $\mathfrak{F}$-group if and only if $G$ is a $\pi$-group and, for each $p$-chief factor $U / V$ of $G, G / C_{G}(U / V) \in \mathfrak{f}(p)$.

It is clear that $\mathfrak{F}$ satisfies condition (S1) of the definition. Now let $G=D \rtimes M$ be a semiprimitive Chernikov group with $D$ a $p$-group. First suppose that $G \in \mathfrak{F}$; then $M \cong$ $G / D \in \mathfrak{F}$. If $H$ is a finite subgroup of $G$ containing $M$ and $U / V$ is a $p$-chief factor of $H$ with $U \leqslant D \cap H$, then $U / V$ is a $p$-chief factor of $G$ and $H / C_{H}(U / V) \cong G / C_{G}(U / V) \in \mathfrak{f}(p)$. It follows that $H \in \mathbb{F}$ and so $G$ is the union of an ascending chain of finite $\mathfrak{F}$-groups. Conversely, suppose that $G=\bigcup_{i=1}^{\infty} H_{i}$ with $H_{i} \in \mathfrak{F}$. We may assume that $M \leqslant H_{i}$ and so $M \cong G / D \cong H_{i} / D \cap H_{i} \in \mathfrak{F}$. If $U / V$ is a $p$-chief factor of $G$ with $U \leqslant D$ then $U$ is finite, since $D$ is divisibly irreducible. Therefore $U \leqslant H_{i}$, for some $i$, and, since $D H_{i}=G, U / V$ is 
a $p$-chief factor of $H_{i}$. Therefore $G / C_{G}(U / V) \cong H_{i} / C_{H_{i}}(U / V) \in \mathfrak{f}(p)$ and so $G \in \mathfrak{F}$. Thus condition (S2) is satisfied.

Finally, suppose that $G$ is an $\mathscr{U} \mathcal{S}^{*}$-group with every finite primitive and infinite semiprimitive homomorphic image in $\mathfrak{F}$. Then by the residual closure property of $\mathfrak{F}$, we have $G / \mu(G) \in \mathfrak{F}$. Since $\mathfrak{F}$ is saturated, it follows that $G \in \mathfrak{F}$.

\subsection{Gaschütz $\Omega$-subgroups}

Let $\Omega$ be a subset of $\mathbb{N}$ such that $\Omega^{\prime}=\mathbb{N} \backslash \Omega$ is multiplicatively closed. A finite soluble group is then defined to be an $\Omega$-group if the index of each maximal subgroup is in the set $\Omega$.

For $\mathfrak{U}^{*}$-groups the definition of an $\Omega$-group is slightly more complicated as we have to consider major subgroups and allow for a semiprimitive $\Omega$-group being the union of finite $\Omega$-subgroups.

Definition 5.1. An $\mathfrak{A S}^{*}$-group $G$ is an $\Omega$-group if, for every major subgroup $M$ of $G$, there are subgroups $U$ and $V$ containing $M$ with $V$ maximal in $U$ and $|U: V| \in \Omega$.

Of course, if $M$ is a maximal subgroup of $G$ then this condition simply says that $|G: M| \in \Omega$ and so for finite soluble groups we have the usual definition. If $M$ is a nonmaximal major subgroup of $G$, then there is an ascending chain of subgroups

$$
M \leqslant V_{1}<U_{1} \leqslant V_{2}<U_{2} \leqslant \cdots<G
$$

such that $V_{i}$ is maximal in $U_{i}$ and $\left|U_{i}: V_{i}\right| \in \Omega$.

It is not immediately clear that the class of $\Omega$-groups forms a Schunck class of $\mathfrak{A S}^{*}$ groups and to prove this we require the existence in finite soluble groups of Gaschütz $\Omega$-subgroups.

Definition 5.2. A Gaschütz $\Omega$-subgroup of the group $G$ is an $\Omega$-subgroup $S$ of $G$ such that $|U: V| \notin \Omega$ whenever $S \leqslant V<U \leqslant G$ and $V$ is maximal in $U$.

Proposition 5.3. [4, Theorem III.10] The finite $\Omega$-groups form a Schunck class of finite soluble groups and the projectors of a finite soluble group $G$ are the Gaschütz $\Omega$-subgroups of $G$.

We make use of this to prove that the $\Omega$-groups form a Schunck class of $\mathfrak{A}^{*}$-groups. It is clear that they form a $Q$-closed class and, since the definition only refers to finite primitive and infinite semiprimitive images of $G$, condition (S3) will also be satisfied. The only problem arises from the infinite semiprimitive groups and this is dealt with in the following result.

Lemma 5.4. Let $G=D \rtimes M$ be an infinite semiprimitive Chernikov group. Then $G$ is an $\Omega$-group if and only if $G$ is the union of ascending chain of finite $\Omega$-subgroups. 
Proof. First, suppose that $G=\bigcup_{i=1}^{\infty} H_{i}$ with $H_{i}$ a finite $\Omega$-group. If $L$ is a maximal subgroup of $G$, then $L \geqslant D$ and, since $G / D \cong H_{i} / D \cap H_{i}$, for some $i, G / D$ is an $\Omega$-group and so $|G: L| \in \Omega$. If $L$ is any non-maximal major subgroup of $G$ then $L$ is finite and so $L<H_{i}$, for some $i$. There is a subgroup $K_{i}$ with $L \leqslant K_{i}<H_{i}$ and $K_{i}$ maximal in $H_{i}$. Since $H_{i}$ is an $\Omega$-group, $\left|H_{i}: K_{i}\right| \in \Omega$. Hence $G$ is an $\Omega$-group.

Conversely, suppose that $G$ is an $\Omega$-group; then $G / D$ is a finite $\Omega$-group. Also there is an ascending chain of subgroups,

$$
M \leqslant V_{1}<U_{1} \leqslant V_{2}<U_{2} \leqslant \cdots<G
$$

with $V_{i}$ maximal in $U_{i}$ and $\left|U_{i}: V_{i}\right| \in \Omega$. Note that if $K_{i}$ is the core of $V_{i}$ in $U_{i}$ then $U_{i} / K_{i}$ is an $\Omega$-group. Choose subgroups $X_{i}$ of $U_{i}$ such that

$$
M \leqslant X_{1} \leqslant X_{2} \leqslant \cdots
$$

and $X_{i}$ is a maximal $\Omega$-subgroup of $U_{i}$. Since $G=D M$ we have $D X_{i}=G$ and hence $\left(D \cap U_{i}\right) X_{i}=U_{i}$. It follows from Lemma 4.7 that $X_{i}$ is a projector of $U_{i}$ and hence $K_{i} X_{i}=U_{i}$. Therefore $X_{i}>X_{i} \cap V_{i} \geqslant X_{i} \cap U_{i-1} \geqslant X_{i-1}$ and the chain (*) is a properly ascending chain. Let $X=\bigcup_{i=1}^{\infty} X_{i}$; then $X$ is an infinite subgroup of $G$ and $D X=G$. Therefore $D \cap X$ is an infinite normal subgroup of $G$ and, since $D$ is divisibly irreducible $D \cap X=D$. Therefore $G=X=\bigcup_{i=1}^{\infty} X_{i}$ is the union of an ascending chain of finite $\Omega$-subgroups.

It follows that the class of $\Omega$-groups forms a Schunck class of $\mathscr{U} \Im^{*}$-groups and hence every $\mathfrak{A}^{*}$-group has a conjugacy class of projectors associated with this class. We call these $\Omega$-projectors and the existence and conjugacy of Gaschütz $\Omega$-subgroups is just the following characterization of $\Omega$-projectors.

Proposition 5.5. Let $G \in \mathfrak{U S}^{*}$; then the $\Omega$-projectors of $G$ are the Gaschütz $\Omega$ subgroups of $G$.

Proof. Let $X$ be an $\Omega$-projector of $G$ and let $U, V$ be subgroups containing $X$ with $V$ maximal in $U$. Consider the finite primitive group $U / V_{U}$. By Corollary $4.8, X$ is an $\Omega$-projector of $U$ and so $X V_{U} / V_{U}$ is an $\Omega$-projector of $U / V_{U}$. Since $V \geqslant X V_{U}$, it follows from the finite case that $|U: V| \notin \Omega$ and so $X$ is a Gaschütz $\Omega$-subgroup of $G$.

Conversely, let $S$ be a Gaschütz $\Omega$-subgroup of $G$. Then $S N / N$ is clearly a Gaschütz $\Omega$-subgroup of $G / N$ for any $N \triangleleft G$ and so it is sufficient to observe that $S$ is a maximal $\Omega$-subgroup of $G$. Suppose $S$ is properly contained in an $\Omega$-subgroup $X$; then $S$ is contained in a major subgroup of $X$. By definition of a Gaschütz $\Omega$-subgroup, every maximal link above $S$ has index in $\Omega^{\prime}$ and so every maximal link between $M$ and $X$ has index in $\Omega^{\prime}$, contrary to $X$ being an $\Omega$-group. Therefore $S$ is a maximal $\Omega$-subgroup of $G$ and hence is an $\Omega$-projector.

This result would of course be more satisfactory if we could define $\Omega$-groups so that every maximal link above a major subgroup had index in the set $\Omega$. However the following construction shows that this is not possible.

The first part of this construction is based on an example due to Huppert [6, Aufgabe 15 on p. 715]. Let $X=\left\langle x_{1}, x_{2}, x_{3}\right\rangle$ and $Y=\left\langle y_{1}, y_{2}\right\rangle$ be elementary abelian 2-groups of 
orders $2^{3}, 2^{2}$, respectively, and let $z=\left\langle z_{i j} ; i=1,2,3, j=1,2\right\rangle$ be elementary abelian of order $2^{6}$. Then we can form a group $P$ of order $2^{11}$ generated by $X$ and $Y$ in which $Z$ is central and $\left[x_{i}, y_{j}\right]=z_{i j}$.

Now $P$ has an automorphism $\alpha$ of order 21 which induces automorphisms $\alpha_{1}$ of order 7 on $X$ and $\alpha_{2}$ of order 3 on $Y$. Thus $[x, y]^{\alpha}=\left[x^{\alpha_{1}}, y^{\alpha_{2}}\right]$ and $\alpha$ induces an automorphism of order 21 on $Z$. If one considers $X \cong G F\left(2^{3}\right)$ and $\alpha_{1}$ being multiplication by a primitive element and similarly for the $\alpha_{2}$ acting on $Y \cong G F\left(2^{2}\right)$, then $Z \cong G F\left(2^{3}\right) \otimes G F\left(2^{2}\right) \cong$ $G F\left(2^{6}\right)$ and $\alpha_{1} \otimes \alpha_{2}$ generates $G F\left(2^{6}\right)$ over $G F(2)$. Therefore $Z$ is an irreducible $\langle\alpha\rangle$-module.

Form the group $H=P \rtimes\langle\alpha\rangle$ so that $|H|=2^{11} .3 .7$ and let $A=Z X$ and $F=Y\langle\alpha\rangle$. Then $A$ is a $G F(2) F$-module and $A$ is indecomposable. For, if $B$ is a proper nontrivial $F$ submodule of $A$ other than $Z$, then as $\langle\alpha\rangle$-modules $A=B \oplus Z$ and $B \cong \mathfrak{X}$. But $B+X<A$ and since $A / B \cong Z$ is irreducible as an $\langle\alpha\rangle$-module we must have $B=X$. However, $X$ is not an $F$-submodule. Therefore $A$ is an indecomposable $G F(2) F$-module, an extension of the irreducible $Z$ of dimension 6 by $A / Z$ of dimension 3 .

Since the socle of $A$ is irreducible, $A$ can be embedded in a projective indecomposable $G F(2) F$-module $D_{1}$ [7, Lemma VII.10.4] and Soc $D_{1} \cong \operatorname{Soc} A$ has dimension 6. Since $A \leqslant D_{1}, D_{1}$ has an irreducible factor of dimension 3. [In fact, there are just four projective indecomposable modules with socles being isomorphic to $G F(2), P / A$ of dimension $2, A / Z$ of dimension 3 and $Z$ of dimension 6 . The principal block consists of the first two of these and so $D_{1}$ only has composition factors of dimensions 3 and 6.]

The projective indecomposable $G F(2) F$-module $D_{1}$ is also injective [7, Theorem VII.7.8] and so there is an injective $\mathbb{Z} F$-module $D$ such that $\Omega_{1}(D) \cong D_{1}[5$, Theorem 4.1].

By [5, Lemma 2.2], $D$ is a sum of divisibly irreducible submodules and so contains a divisibly irreducible submodule $A$ containing infinitely many composition factors of dimension 3. But also $A D_{n} / D_{n}$ is non-trivial and so contains $\operatorname{Soc}\left(D / D_{n}\right)=S_{n} / D_{n}$, which is irreducible of dimension 6 . Therefore $A$ also has infinitely many composition factors $A \cap S_{n} / A \cap D_{n}$ of dimension 6 .

Now form $G=A \rtimes F ; G$ is a semiprimitive Chernikov group with $F$ being a major subgroup. Let $\Omega=\left\{3,7,2,2^{2}, 2^{3}\right\}$; since $A$ has infinitely many composition factors of order $2^{3}$ it follows that $G$ is an $\Omega$-group even though there are maximal links of index $2^{6}$ above every nonmaximal major subgroup.

\section{REFERENCES}

1. K. Doerk and T. O. Hawkes, Finite soluble groups (de Gruyter, 1992).

2. A. D. Gardiner, B. Hartley and M. J. Tomkinson, Saturated formations and Sylow structure in locally finite groups, J. Algebra 17 (1971), 177-211.

3. W. GASCHOTZ, Zur Theorie der endlichen aufösbaren Gruppen, Math. Z. 80 (1963), 300-305.

4. W. GASCHÜtZ, Lectures on subgroups of Sylow type in finite soluble groups (Australian National University, 1979).

5. B. Hartley, A dual approach to Cernikov modules, Math. Proc. Cambridge Philos. Soc. 82 (1977), 215-239.

6. B. HuPpert, Endliche Gruppen I (Springer, 1968). 
7. B. HupPert and N. Blackburn, Finite groups II (Springer, 1982).

8. H. SchunCk, 5-Untergruppen in endlichen auflösbaren Gruppen. Math. Z. 97 (1967), 326-330.

9. M. J. Tomkinson, A Frattini-like subgroup, Math. Proc. Cambridge Philos. Soc. 77 (1975), 247-257.

10. M. J. Tomkinson, Major subgroups of nilpotent-by-finite groups, Ukrain. Mat. $\mathrm{Zh} .44$ (1992), 853-856.

Department of Mathematics

UNIVERSITY OF GLASGOW

GLASGOW G12 8QW

SCOTLAND 